\title{
Evaluación antropométrica de los preescolares de la zona urbana en Cuenca, Ecuador
}

\author{
Ruth Díaz-Granda, Lourdes Huiracocha \\ Facultad de Ciencias Médicas, Universidad de Cuenca, Cuenca, Ecuador. \\ Autor para correspondencia: ruth.diazg@ucuenca.edu.ec \\ Fecha de recepción: 25 de septiembre de 2015 - Fecha de aceptación: 31 de octubre 2015
}

\begin{abstract}
RESUMEN
Se realizó un estudio transversal que incluyó 577 preescolares que acudieron a control del niño sano. Se excluyó a niños con patología. Se estudió peso, talla e IMC, según subgrupos de edad y sexo. Se obtuvieron frecuencias absolutas y relativas para las variables cualitativas; promedio y DE para las variables cuantitativas. Se determinó la comparabilidad de grupos según sexo $\left(\chi^{2}, p>0.05\right)$ y edad (U de Mann-Whitney, p>0.05). Se correlacionó y graficó: peso/edad, talla/edad e IMC/edad. La antropometría promedio para el grupo fue: peso $12.006 \mathrm{~kg} \pm 2.636$; talla $86.3 \mathrm{~cm} \pm 10.0$; IMC 16.1 $\mathrm{kg} \mathrm{m}^{-2} \pm 1.7$; los varones presentaron medias mayores. El estado nutricional del grupo fue normal según la relación: peso/edad en $90.8 \%$, talla/edad $75.6 \%$, IMC/edad $77.5 \%$. Presentaron baja talla/edad $24.3 \%$ y sobrepeso/obesidad $4.7 \%$. Los varones tuvieron mayor frecuencia de bajo peso severo, bajo peso, baja talla severa, talla baja, riesgo de sobrepeso y sobrepeso. En los dos sexos, la correlación peso/edad, talla/edad y peso/talla fue >0.8; la correlación IMC/edad fue <-0.3. Se concluye que estuvieron presentes todos los tipos de malnutrición. El déficit de talla/edad fue el problema nutricional más importante. La correlación peso/edad, talla/edad y peso/talla fue positiva y alta. El mejoramiento de las condiciones de vida de los países latinoamericanos no evidenció mejora en las condiciones nutricionales de los niños estudiados.
\end{abstract}

Palabras clave: Antropometría, peso, talla, IMC, correlación, niños, preescolares, servicios públicos de atención de salud.

\begin{abstract}
A cross-sectional study involving 577 preschool children attending regular well-baby check-ups was performed. Children with pathological deviations were excluded. Weight, height and BMI were monitored, according to age subgroups and gender. Absolute and relative frequencies were obtained for the qualitative variables; the average and the SD for quantitative variables. Comparability of groups was determined by sex $\left(\chi^{2}, p>0.05\right)$ and age (Mann-Whitney, $\left.p>0.05\right)$. Correlations were defined and plotted between weight/age, height/ age and BMI/age. The average anthropometry for the group was: weight $12,006 \pm 2.636 \mathrm{~kg}$; height $10.0 \pm 86.3 \mathrm{~cm}$; BMI $16.1 \mathrm{~kg} \mathrm{~m}^{-2} \pm 1.7$; men had higher averages. The nutritional status of the group was normal according to the ratio weight/age at $90.8 \%$, height/age at $75.6 \%, \mathrm{BMI} / \mathrm{age}$ at $77.5 \%$. They presented low height/age and $24.3 \%$ overweight/ obesity $4.7 \%$. Males had a higher frequency of severely underweight, underweight, severely stunted, stunting, overweight risk and overweight. The correlation weight/age, height/age and weight/height in both sexes was $>0.8$; the correlation BMI/age was $<-0.3$. The survey demonstrated that all types of malnutrition were present, and the deficit height/age seems the most important nutritional bottle neck. Correlation weight/age, height/age and weight/height were positive and high. Improving the living conditions of Latin American countries showed no improvement in the nutritional status of the children surveyed.
\end{abstract}

Keywords: Anthropometry, weight, height, BMI, correlation, children, preschool, public health care services. 


\section{INTRODUCCIÓN}

El crecimiento es un proceso dinámico y complejo que se inicia con la fertilización del óvulo; consiste en una cadena sistemática de fenómenos celulares caracterizados por multiplicación e hipertrofia que confluyen hacia aspectos fisiológicos y morfológicos; obedece a factores intrínsecos como los genéticos, sexo, actividad hormonal, enfermedades y raza; así como a los factores ambientales entre los que están la nutrición, las condiciones sociales, el ejercicio, el clima, etc. El crecimiento no es uniforme; a lo largo del tiempo se alternan unos periodos intensos con otros de calma relativa (Crecimiento y desarrollo, 2003). Además, los varones, en general, crecen más que las mujeres.

El crecimiento está íntimamente vinculado a parámetros físicos como peso, talla, índice de masa corporal y circunferencias corporales que reflejan, en cierto modo, la composición corporal. Estos parámetros son de gran importancia para la salud individual y colectiva porque evalúan el bienestar general de los niños, de las comunidades en las que ellos viven, así como los avances sanitarios (Cossio-Bolaños et al., 2011). El crecimiento puede ser investigado en forma sencilla, práctica, barata y no invasiva a través de la determinación de las medidas antropométricas (Arroyo et al., 2010). Las referencias del crecimiento de lactantes y niños pequeños son instrumentos muy utilizados en salud pública y en la práctica clínica (Atalah, 2007) porque permiten evaluar el estado nutricional (Gutiérrez-Gómez et al., 2009), facilitan conocer el estado de salud (León et al., 2010), realizar el diagnóstico de alteraciones nutricionales y planificar las medidas preventivas (Angarita et al., 2001). Para el estudio de la situación nutricional el enfoque más utilizado se refiere a la combinación de los indicadores peso y talla (Velásquez et al., 2008) valorados de acuerdo con la edad y el sexo.

En el Ecuador, según los estudios de la CEPAL, desde 1986 al 2006 la desnutrición global (déficit de peso/edad) de los niños de 12 a 59 meses disminuyó de 16.5 a $8.6 \%$, y fue mayor que en Bolivia, Paraguay y Perú (Martínez \& Fernández, 2009). Hacia 1991, la desnutrición global había llegado a 14.8\%; y en 1999 la desnutrición crónica (déficit de talla/edad) fue 27.1\% (Martínez \& Fernández, 2006). La información proporcionada por ENSANUT-ECU refiere que en el año 2012, en niños menores de 60 meses, el retardo de talla (talla/edad <-2DE) fue $25.3 \%$, el bajo peso (peso/edad $<-2 \mathrm{DE}$ ) $6.4 \%$, la emaciación (peso/talla <-2DE) $2.4 \%$ y el sobrepeso/obesidad (IMC para la edad $>+2 \mathrm{DE}$ ) $8.6 \%$ (Freire et al., 2013).

En las últimas décadas, la población latinoamericana ha logrado avances importantes en salud, educación y vivienda. El Ecuador, como país latinoamericano, debe haberse beneficiado de los cambios de la realidad socioeconómica y de salud de la región. Teniendo en cuenta, por una parte, que los menores de cinco años constituyen un grupo prioritario en la atención de salud, y representan alrededor del 10\% de la población nacional (INEC, 2013); y por otro lado, que el crecimiento es uno de los principales parámetros para la evaluación de la situación de salud individual y colectiva; se consideró importante determinar la condición nutricional actual de los niños menores de cinco años con el fin conocer los progresos con respecto al pasado y contar con información para la planificación de estrategias de salud dirigidas a disminuir la desnutrición y limitar el avance del sobrepeso y la obesidad.

Por esta razón, se planteó la realización de un estudio que determine las condiciones antropométricas de peso, talla e índice de masa corporal de los niños y niñas de 12 a 59 meses de edad que asistieron a los servicios de salud del eje urbano y área de influencia de la ciudad de Cuenca en el Ecuador durante el año 2013; así como establecer la correlación antropométrica según el sexo y la edad. De modo que los objetivos específicos fueron: (1) Describir las medidas antropométricas (peso, talla e índice de masa corporal) según edad y sexo en los niños de uno a cinco años que asistieron a los servicios de salud del eje urbano y área de influencia de la ciudad de Cuenca en el Ecuador; y (2) Determinar la correlación de las medidas antropométricas (peso, talla e índice de masa corporal) según el sexo y la edad de los niños que participaron en el estudio. El estudio se realizó en el año 2013, sobre la base poblacional que acudió a 18 centros públicos de salud localizados en Cuenca y sus alrededores. 


\section{MATERIAL Y MÉTODOS}

Este estudio formó parte del Proyecto de Dificultades Alimentarias realizado en Cuenca con el aval de la Universidad de Cuenca.

2.1. Tipo de estudio. Se trató de un estudio epidemiológico transversal realizado en 18 centros de salud pública del eje urbano y área de influencia inmediata de la ciudad de Cuenca: Centro de Salud $\mathrm{N}^{\mathrm{o}} 1$ (Pumapungo), Paraíso, Machángara, Totoracocha, Centro de Salud No 2 (José María Astudillo Regalado), Terminal Terrestre, Barrial Blanco, Uncovía, Cebollar, San Pedro del Cebollar, Centro de Salud No 3 (Nicanor Merchán), Tomebamba, Turi, Centro de Salud No 4 (Carlos Elizalde), Baños, Virgen del Milagro, San Joaquín, Sayausí; durante enero a junio del 2013.

2.2. Población y muestra. La población estuvo constituida por niños y niñas de 12 a 59 meses de vida que asistieron a los 18 centros de salud pública. Se seleccionó una muestra no probabilística, secuencial según orden de llegada a los servicios; calculada para una población infinita, con nivel de confianza de $95 \%$, precisión del $3 \%$, proporción de niños menores de 5 años de $10 \%$ y con una proporción esperada de pérdidas de 4\%, obteniéndose un tamaño muestral de 576 niños. La unidad de observación y análisis fue individual. Los criterios de inclusión fueron: niños o niñas, con edades entre 12 y 59 meses cumplidos, que asistieron a control del niño sano en una de las 18 unidades en las que se realizó el estudio, cuyos familiares firmaron el consentimiento informado y los niños mostraron su disposición a participar. Se excluyó a los niños que presentaron patologías.

2.3. Variables de estudio. Las variables estudiadas fueron las medidas antropométricas peso, talla e índice de masa corporal. Las mismas que varían con la edad y el sexo. El peso se definió como la fuerza ejercida por el cuerpo del niño o niña, se determinó de acuerdo a las normas establecidas por la OMS (2008) en una balanza de palancas, se expresó en kilogramos y sus fracciones, con error de $0.1 \mathrm{~kg}$. La talla se concibió como la longitud desde la coronilla hasta la planta de los pies; se determinó de acuerdo a las normas establecidas por la OMS (2008), se expresó en centímetros con un error de $0.1 \mathrm{~cm}$. El índice de masa corporal se calculó de acuerdo a la fórmula: peso $(\mathrm{kg}) / \mathrm{talla}^{2}$ $\left(\mathrm{m}^{2}\right)$, con un error de 0.1. La edad se consideró como el tiempo transcurrido desde el día del nacimiento del niño o niña hasta el día de la evaluación, se expresó en meses completos, con error de un mes. El sexo se definió como el fenotipo genital referido por los padres y se catalogó como masculino o femenino. Para determinar la condición nutricional individual en relación a la edad se utilizó las gráficas oficiales de peso, talla e índice de masa corporal según edad y sexo del Ministerio de Salud del Ecuador que están expresadas en puntuaciones respecto a la media; para la posterior clasificación del estado nutricional se siguió los parámetros oficiales recomendados por dicho ministerio; según el escore $Z$ de peso/edad se clasificó en bajo peso severo (<-3), bajo peso $(<-2)$, peso normal $(-2 y+2)$ y peso alto $(>+2)$; según el escore $Z$ de talla/edad, se clasificó en talla baja severa $(<-3)$, talla baja $(<-2)$, talla normal $(-2 y+3)$ y talla alta $(>+3)$; según el escore $Z$ de IMC/edad, se clasificó en emaciado severo $(<-3)$, emaciado $(<-2)$, normal $(-2 \mathrm{y}+1)$, riesgo de sobrepeso $(>+1)$, sobrepeso (>+2), obesidad (>+3) (MSP-Ecuador, 2011).

2.4. Instrumento y proceso de recolección de la información. Para la recolección de la información se elaboró un formulario individual, que fue sometido a valoración por expertos y a prueba de campo con 30 niños, lo que contribuyó a la unificación de criterios. La recolección de la información y toma de las medidas antropométricas estuvo a cargo de las nutricionistas del Proyecto de Dificultades Alimentarias en Niños Menores de Cinco Años de Edad, quienes previamente fueron entrenadas, y acudieron de lunes a viernes a los centros de salud en los que se realizó el estudio.

2.5 Análisis estadístico. Los datos codificados fueron introducidos en el programa SPSS (versión 22). Se aplicó estadística descriptiva con obtención de frecuencias absolutas y relativas para la variable sexo; frecuencias absolutas y relativas, media y desviación estándar (DE) para la variable edad y cada uno de sus subgrupos. Las variables continuas peso/edad, talla/edad e IMC/edad se categorizaron de manera ordinal y se estableció su frecuencia absoluta y relativa. Se determinó la comparabilidad de los grupos según sexo (prueba de $\chi^{2}, p>0.05$ ) y edad (prueba U de MannWhitney, $\mathrm{p}>0.05$ ). Se determinó según sexo la correlación de peso/edad, talla/edad e IMC/edad con una significancia estadística $<0.05$; y finalmente se graficó la correlación peso/edad, talla/edad, IMC/edad y talla/edad. 


\section{RESULTADOS}

El grupo se conformó con 577 preescolares: 48.9\% (282) de sexo masculino y 51.1\% (295) de sexo femenino (Tabla 1). En el grupo total, la edad osciló entre los 12 y 59 meses, con un promedio de 30.36 \pm 13.53 . Presentaron edades entre: $12-23$ meses $36,9 \%$; $24-35$ meses $27.7 \%$; $36-47$ meses $21.1 \%$ y $48-59$ meses $14.3 \%$. La distribución de frecuencias según edad y sexo se encuentra en la Tabla 1. Las medidas antropométricas promedio, para el grupo total fueron: peso $12.006 \mathrm{~kg} \pm 2.636$; talla $86.3 \mathrm{~cm} \pm 10.0$; índice de masa corporal $16.1 \mathrm{~kg} \mathrm{~m}^{-2} \pm 1.7$. La muestra se dividió en grupos comparables según sexo mediante $\chi^{2}$ con $p>0.59$ (Tabla 1) y edad a través de la prueba $U$ de Mann-Whitney con $p>0.05$ (Tabla 2).

Tabla 1. Distribución según edad y sexo de 577 preescolares que asistieron en el 2013 a los servicios de salud del Ministerio de Salud Pública en Cuenca, Ecuador.

\begin{tabular}{lcccccc}
\hline \multirow{2}{*}{ Edad (meses) } & \multicolumn{2}{c}{ Masculino } & \multicolumn{2}{c}{ Femenino } & \multirow{2}{*}{$\chi^{2}$} & $\mathrm{p}$ \\
\cline { 2 - 5 } & $\mathrm{N}^{\mathbf{o}}$ & $\%(*)$ & $\mathrm{N}^{\mathrm{o}}$ & $\%(*)$ & & 0.732 \\
$12-23$ & 104 & 18.0 & 109 & 18.9 & 0.117 & 0.429 \\
$24-35$ & 75 & 13.0 & 85 & 14.7 & 0.625 & 0.456 \\
$36-47$ & 60 & 10.4 & 62 & 10.7 & 0.033 & 0.856 \\
$48-59$ & 43 & 7.5 & 39 & 6.8 & 0.195 & 0.659 \\
Total & 282 & 48.9 & 295 & 51.1 & 0.293 & 0.588 \\
\hline
\end{tabular}

(*) \% en relación al grupo total.

Tabla 2. Distribución según sexo, edad y medidas antropométricas promedio de los preescolares que asistieron en el 2013 a los servicios de salud del Ministerio de Salud Pública en Cuenca, Ecuador.

\begin{tabular}{lccccc}
\hline \multirow{2}{*}{ Grupo } & \multicolumn{2}{c}{ Masculino } & \multicolumn{2}{c}{ Femenino } & \multirow{2}{*}{$\mathrm{p}$} \\
\cline { 2 - 5 } & Promedio & DE & Promedio & DE & \\
\hline 12-23 meses & & & & & \\
Edad (meses) & 16.7 & 3.5 & 16.2 & 3.4 & 0.400 \\
Peso $(\mathrm{kg})$ & 10.039 & 1.257 & 9.614 & 1.211 & 0.009 \\
Talla $(\mathrm{cm})$ & 77.1 & 4.5 & 75.7 & 4.6 & 0.024 \\
IMC $\left(\mathrm{kg} / \mathrm{m}^{2}\right)$ & 16.9 & 1.7 & 16.7 & 1.5 & 0.525 \\
\hline $24-35$ meses & & & & & \\
Edad $(\mathrm{meses})$ & 29.1 & 3.5 & 28.7 & 3.3 & 0.467 \\
Peso $(\mathrm{kg})$ & 12.119 & 1.579 & 11.394 & 1.525 & 0.000 \\
Talla $(\mathrm{cm})$ & 86.5 & 4.6 & 86.0 & 4.2 & 0.431 \\
IMC $\left(\mathrm{kg} / \mathrm{m}^{2}\right)$ & 16.2 & 1.6 & 15.4 & 1.6 & 0.001 \\
\hline $36-47$ meses & & & & & \\
Edad $(\mathrm{meses})$ & 40.3 & 3.3 & 41.8 & 3.2 & 0.011 \\
Peso $(\mathrm{kg})$ & 13.832 & 2.163 & 13.647 & 1.441 & 0.961 \\
Talla $(\mathrm{cm})$ & 93.6 & 5.3 & 94.1 & 4.0 & 0.289 \\
IMC $\left(\mathrm{kg} / \mathrm{m}^{2}\right)$ & 15.8 & 1.6 & 15.5 & 1.4 & 0.363 \\
\hline $48-59$ meses & & & & & \\
Edad $(\mathrm{meses})$ & 53.6 & 3.4 & 53.2 & 3.4 & 0.625 \\
Peso $(\mathrm{kg})$ & 15.772 & 2.504 & 15.477 & 1.844 & 0.477 \\
Talla $(\mathrm{cm})$ & 100.7 & 7.0 & 101.1 & 4.1 & 0.897 \\
IMC $\left(\mathrm{kg} / \mathrm{m}^{2}\right)$ & 15.6 & 1.3 & 15.1 & 1.2 & 0.083 \\
\hline
\end{tabular}




\begin{tabular}{lccccc}
\hline Todo del grupo & & & & & \\
Edad (meses) & 30.7 & 13.7 & 30.1 & 13.5 & 0.644 \\
Peso $(\mathrm{kg})$ & 12.302 & 2.759 & 11.8749 & 2.5 & 0.022 \\
Talla $(\mathrm{cm})$ & 86.8 & 10.2 & 85.9 & 10.1 & 0.407 \\
$\mathrm{IMC}\left(\mathrm{kg} / \mathrm{m}^{2}\right)$ & 16.3 & 1.7 & 15.9 & 1.6 & 0.002 \\
\hline
\end{tabular}

$\mathrm{DE}=$ desvío estándar.

En la Tabla 2 se presentan las medidas antropométricas y la significancia estadística que se estableció según el sexo tanto para los subgrupos de edad como para todo el grupo. En el subgrupo de 36-47 meses, la edad media de los niños fue diferente a la de las niñas; por lo tanto, las medidas antropométricas de este subgrupo no son comparables según el sexo. En los dos sexos el peso y la talla se incrementaron progresivamente con la edad, mientras que el índice de masa corporal disminuyó. El peso promedio de los niños fue mayor al de las niñas y presentó diferencia significativa en los subgrupos de 12-23 meses, 24-35 meses. La talla media fue significativamente superior en los varones 12-23 meses con relación a las mujeres; en el subgrupo de 24 a 35 meses la talla masculina es superior con $0.5 \mathrm{~cm}$ pero no establece diferencia significativa con la talla femenina; inversamente, en el subgrupo de 48-59 meses la talla de las niñas es mayor, sin diferencia significativa con la de los niños. El IMC promedio fue significativamente mayor para los varones de 24-35 meses con respecto a las mujeres. Sin diferenciar por sexo, en la clasificación según el peso/edad presentaron normalidad 90.8\% (524 niños y niñas), bajo peso $6.6 \%$ (38 niños y niñas), peso bajo severo $1.6 \%$ (9 niños y niñas), peso alto $1.0 \%$ (6 niños y niñas) (Tabla 6). Un porcentaje ligeramente mayor de niñas $(92.2 \%)$ presentó peso normal en relación a los niños (89.4\%); el peso bajo severo fue más frecuente en el sexo masculino (Tabla 3).

Tabla 3. Distribución según sexo y peso/edad de los preescolares que asistieron en el 2013 a los servicios de salud del Ministerio de Salud Pública en Cuenca-Ecuador.

\begin{tabular}{lccccc}
\hline \multirow{2}{*}{ Grupo } & \multicolumn{2}{c}{ Masculino } & \multicolumn{2}{c}{ Femenino } & \multirow{2}{*}{$\mathrm{p}$} \\
\cline { 2 - 5 } & $\mathrm{N}^{\mathrm{o}}$ & $\%$ & $\mathrm{~N}^{\mathrm{o}}$ & $\%$ & 0.292 \\
Nor-23 meses & 93 & 89.4 & 103 & 94.5 & \\
Peso bajo & 7 & 6.7 & 5 & 4.6 & \\
Peso bajo severo & 3 & 2.9 & 0 & 0.0 & \\
Peso alto & 1 & 1.0 & 1 & 0.9 & \\
\hline 24-35 meses & & & & & 0.613 \\
Normal & 69 & 92.0 & 76 & 89.4 & \\
Peso bajo & 5 & 6.7 & 6 & 7.1 & \\
Peso bajo severo & 1 & 1.3 & 1 & 1.2 & \\
Peso alto & 0 & 0.0 & 2 & 2.4 & \\
\hline 36-47 meses & & & & & \\
Normal & 50 & 83.3 & 57 & 91.9 & \\
Peso bajo & 6 & 10.0 & 5 & 8.1 & \\
Peso bajo severo & 3 & 5.0 & 0 & 0.0 & \\
Peso alto & 1 & 1.7 & 0 & 0.0 & \\
\hline 48-59 meses & & & & & \\
Normal & 40 & 93.1 & 36 & 92.3 & \\
Peso bajo & 1 & 2.3 & 3 & 7.7 & \\
Peso bajo severo & 1 & 2.3 & 0 & 0.0 & \\
Peso alto & 1 & 2.3 & 0 & 0.0 & \\
\hline
\end{tabular}




\begin{tabular}{lccccc}
\hline Todo del grupo & & & & & 0.116 \\
Normal & 252 & 89.4 & 272 & 92.2 & \\
Peso bajo & 19 & 6.7 & 19 & 6.4 & \\
Peso bajo severo & 8 & 2.8 & 1 & 0.3 & \\
Peso alto & 3 & 1.1 & 3 & 1.0 & \\
\hline
\end{tabular}

El peso, la talla e IMC promedio fueron mayores en los niños que en las niñas. La relación talla/edad fue normal en un $75.6 \%$ (436 niños); presentaron talla baja 20.8 (120 niños), talla baja severa $3.5 \%$ (20 niños), talla alta 0.2 (1 niño). La frecuencia de talla normal fue mayor en las niñas (79.0\%) que en niños (72.0\%); la talla baja y talla baja severa tuvieron mayor frecuencia en los niños, en los distintos subgrupos de edad. En las niñas con el incremento de la edad se elevó el porcentaje de normalidad. La frecuencia de talla baja y talla baja severa se redujo en el grupo de 48-59 meses (Tabla 4).

Tabla 4. Distribución según sexo y talla/edad de los preescolares que asistieron en el 2013 a los servicios de salud del Ministerio de Salud Pública en Cuenca-Ecuador.

\begin{tabular}{lccccc}
\hline \multirow{2}{*}{ Grupo } & \multicolumn{2}{c}{ Masculino } & \multicolumn{2}{c}{ Femenino } & \multirow{2}{*}{$\mathrm{p}$} \\
\cline { 2 - 5 } 12-23 meses & $\mathrm{N}^{\mathrm{o}}$ & $\%$ & $\mathrm{~N}^{\mathrm{o}}$ & $\% .227$ \\
Normal & 71 & 68.3 & 84 & 77.1 & \\
Talla baja & 24 & 23.1 & 22 & 20.2 & \\
Talla baja severa & 8 & 7.7 & 3 & 2.8 & \\
Talla alta & 1 & 1.0 & 0 & 0.0 & \\
\hline 24-35 meses & & & & & 0.712 \\
Normal & 56 & 74.7 & 66 & 77.6 & \\
Talla baja & 19 & 25.3 & 19 & 22.4 & \\
Talla baja severa & 0 & 0.0 & 0 & 0.0 & \\
Talla alta & 0 & 0.0 & 0 & 0.0 & \\
\hline 36-47 meses & & & & & \\
Normal & 40 & 66.7 & 49 & 79.0 & \\
Talla baja & 14 & 23.3 & 11 & 17.7 & \\
Talla baja severa & 6 & 10.0 & 2 & 3.2 & \\
Talla alta & 0 & 0.0 & 0 & 0.0 & \\
\hline 48-59 meses & & & & & \\
Normal & 36 & 83.7 & 34 & 87.2 & \\
Talla baja & 6 & 14.0 & 5 & 12.8 & \\
Talla baja severa & 1 & 2.3 & 0 & 0.0 & \\
Talla alta & 0 & 0.0 & 0 & 0.0 & \\
\hline Todo del grupo & 1 & & & & \\
Normal & 63 & 22.3 & 57 & 19.3 & \\
Talla baja & 15 & 5.3 & 5 & 1.7 & \\
Talla baja severa & 0.4 & 0 & 0.0 & \\
Talla alta & & & & \\
\hline
\end{tabular}

El IMC/edad fue normal en 77.5\% (447 niños); presentaron emaciación 1.4\% (8 niños), emaciación severa $0.2 \%$ (1 niño), riesgo de sobrepeso $16.3 \%$ (94 niños), sobrepeso $4.0 \%$ (23 niños) y obesidad $0.7 \%$ (4 niños). Las niñas $(80.3 \%$ ) presentaron mayor frecuencia de normalidad que los varones (74.4\%); el sobrepeso fue más frecuente en los varones en los distintos subgrupos de edad, la emaciación severa, emaciación y obesidad fueron poco frecuentes tanto en varones como en mujeres (Tabla 5). El peso/edad (Tabla 3), la talla/edad (Tabla 4) e IMC/edad (Tabla 5) no evidenciaron diferencias estadísticas 
significativas en relación al sexo, en los diferentes subgrupos etarios ni en el grupo total. La situación nutricional de todo el grupo se ha resumido en la Tabla 6, en la misma que además se han registrado los indicadores de malnutrición según las encuestas nacionales DANS (1986), ENDEMAIN (2014) y ENSANUT-ECU (2011-2013) con el objeto de comparar la modificación del déficit de peso/edad, déficit de talla/edad y sobrepeso-obesidad en las últimas tres décadas.

Tanto en las niñas como en los niños, las correlaciones peso/edad, talla/edad y peso/talla fueron muy altas y directas (>0.8). Mientras que la correlación del IMC/edad fue inversa y baja. La correlación talla/edad fue la de mayor grado en los dos sexos. En los niños la edad predijo la talla en 79\%; y en las niñas la edad predijo la talla en $87.4 \%$. En los varones, la edad predijo el peso en un $60.9 \%\left(\mathrm{r}^{2}=0.609\right)$; y en las mujeres, la edad predijo el peso en $71.9 \%\left(\mathrm{r}^{2}=0.719\right)$. En el análisis de la relación talla/peso, se observó que en el sexo masculino, la talla predijo el $78.7 \%$ del peso; y en el sexo femenino, la talla predijo el $78.7 \%$ del peso (Tabla 7).

Tabla 5. Distribución según sexo e IMC/edad de los preescolares que asistieron en el 2013 a los servicios de salud del Ministerio de Salud Pública en Cuenca-Ecuador.

\begin{tabular}{|c|c|c|c|c|c|}
\hline \multirow{2}{*}{ Grupo } & \multicolumn{2}{|c|}{ Masculino } & \multicolumn{2}{|c|}{ Femenino } & \multirow{2}{*}{$\mathrm{p}$} \\
\hline & $\mathrm{N}^{\mathrm{o}}$ & $\%$ & $\mathrm{~N}^{\mathrm{o}}$ & $\%$ & \\
\hline $12-23$ meses & & & & & 0.890 \\
\hline Normal & 77 & 74.0 & 80 & 73.4 & \\
\hline Riesgo de sobrepeso & 20 & 19.2 & 22 & 20.2 & \\
\hline Sobrepeso & 6 & 5.8 & 5 & 4.6 & \\
\hline Obesidad & 1 & 1.0 & 1 & 0.9 & \\
\hline Emaciado & 0 & 0.0 & 1 & 0.9 & \\
\hline 24-35 meses & & & & & 0.075 \\
\hline Normal & 51 & 78.0 & 72 & 84.7 & \\
\hline Riesgo de sobrepeso & 16 & 21.3 & 7 & 8.2 & \\
\hline Sobrepeso & 5 & 6.7 & 2 & 2.4 & \\
\hline Obesidad & 0 & 0.0 & 1 & 1.2 & \\
\hline Emaciado & 3 & 4.0 & 2 & 2.4 & \\
\hline Emaciado severo & 0 & 0.0 & 1 & 1.2 & \\
\hline $36-47$ meses & & & & & 0.533 \\
\hline Normal & 45 & 75.0 & 51 & 82.3 & \\
\hline Riesgo de sobrepeso & 10 & 16.7 & 9 & 14.5 & \\
\hline Sobrepeso & 4 & 6.7 & 1 & 1.6 & \\
\hline Obesidad & 0 & 0.0 & 0 & 0.0 & \\
\hline Emaciado & 1 & 1.7 & 1 & 1.6 & \\
\hline $48-59$ meses & & & & & 0.627 \\
\hline Normal & 37 & 86.1 & 34 & 87.2 & \\
\hline Riesgo de sobrepeso & 5 & 11.6 & 5 & 12.8 & \\
\hline Sobrepeso & 1 & 2.3 & 0 & 0.0 & \\
\hline Obesidad & 0 & 0.0 & 0 & 0.0 & \\
\hline Emaciado & 0 & 0.0 & 0 & 0.0 & \\
\hline Todo del grupo & & & & & 0.398 \\
\hline Normal & 210 & 74.4 & 237 & 80.3 & \\
\hline Riesgo de sobrepeso & 51 & 18.1 & 43 & 14.6 & \\
\hline Sobrepeso & 15 & 5.3 & 8 & 2.7 & \\
\hline Obesidad & 2 & 0.7 & 2 & 0.7 & \\
\hline Emaciado & 4 & 1.4 & 4 & 1.4 & \\
\hline Emaciado severo & 0 & 0.0 & 1 & 0.3 & \\
\hline
\end{tabular}


Tabla 6. Condición nutricional de 577 preescolares que asistieron en el 2013 a los servicios de salud del Ministerio de Salud Pública en Cuenca, Ecuador comparada con las encuestas nacionales.

\begin{tabular}{|c|c|c|c|c|c|}
\hline \multirow[t]{2}{*}{ Medida antropométrica } & \multirow{2}{*}{$\begin{array}{c}\text { DANS } \\
\text { Ecuador, } 1986 \\
\%\end{array}$} & \multirow{2}{*}{$\begin{array}{c}\text { ENDEMAIN } \\
\text { Azuay, } 2004 \\
\%\end{array}$} & \multirow{2}{*}{$\begin{array}{l}\text { ENSANUT-ECU } \\
\text { Ecuador, } 2012 \\
\%\end{array}$} & \multicolumn{2}{|c|}{$\begin{array}{l}\text { Díaz \& Huiracocha } \\
\text { Cuenca, } 2013\end{array}$} \\
\hline & & & & $\mathrm{N}^{\mathrm{o}}$ & $\%$ \\
\hline \multicolumn{6}{|l|}{ Pesoledad } \\
\hline Normal & & & & 524 & 90.8 \\
\hline Peso bajo & 12.8 & 8.3 & 6.4 & 38 & 6.6 \\
\hline Peso bajo severo & & 0.6 & & 9 & 1.6 \\
\hline Peso alto & & & & 6 & 1.0 \\
\hline \multicolumn{6}{|l|}{ Talla/edad } \\
\hline Normal & & & & 436 & 75.6 \\
\hline Talla baja & 40.2 & 20.1 & 25.3 & 120 & 20.8 \\
\hline Talla baja severa & & 11.2 & & 20 & 3.5 \\
\hline Talla alta & & & & 1 & 0.2 \\
\hline \multicolumn{6}{|l|}{ IMC/edad } \\
\hline Normal & & & & 447 & 77.5 \\
\hline Riesgo de sobrepeso & & & 21.6 & 94 & 16.3 \\
\hline Sobrepeso/obesidad & 4.2 & & 8.6 & 27 & 4.7 \\
\hline Emaciado & 2.4 & 0.9 & 2.4 & 8 & 1.4 \\
\hline Emaciado severo & & 0.0 & 0.0 & 1 & 0.2 \\
\hline
\end{tabular}

Las encuestas DANS, ENDEMAIN y ENSANUT definen al emaciado según peso/talla <-2 DE

Fuente: Freire et al. (2013). Resumen Ejecutivo. Tomo 1. Encuesta Nacional de Salud y Nutrición del Ecuador. ENSANUT-ECU 2011-2013. Ministerio de Salud Pública/Instituto Nacional de Estadística y Censos. Quito,

Ecuador. Descargado de www.unicef.org/ecuador/ENSANUT_2011-2013_tomo_1.pdf. Informe final

ENDEMAIN 2004. Parte 2. 2014. CEPAR. Descargado de http://microdata.worldbank.org/

index.php/catalog/979.

Tabla 7. Correlaciones antropométricas según edad y sexo de 577 preescolares que asistieron en el 2013 a los servicios de salud del Ministerio de Salud Pública en Cuenca, Ecuador.

\begin{tabular}{lcccc}
\hline \multirow{2}{*}{ Correlación } & \multicolumn{2}{c}{ Masculino } & \multicolumn{2}{c}{ Femenino } \\
\cline { 2 - 5 } & $\mathrm{r}$ & $\mathrm{r}^{2}$ & $\mathrm{r}$ & $\mathrm{r}^{2}$ \\
\hline Peso/edad & $0.805^{* *}$ & 0.609 & $0.860^{* *}$ & 0.719 \\
Talla/edad & $0.903^{* *}$ & 0.790 & $0.940^{* *}$ & 0.874 \\
IMC/edad & $-0.332^{* *}$ & 0.100 & $-0.406^{* *}$ & 0.135 \\
Peso/talla & $0.899^{* *}$ & 0.787 & $0.915^{* *}$ & 0.812 \\
\hline *r con $\mathrm{p}<0.001$. & & & &
\end{tabular}

\section{DISCUSIÓN}

Ante los cambios en las condiciones de vida de los países latinoamericanos, se consideró necesario establecer la condición nutricional de niños y niñas de 12 a 59 meses en la ciudad de Cuenca, en el Ecuador. Los valores promedio de peso y talla, tanto en varones como en mujeres en cada uno de los subgrupos etarios, se ubicaron dentro del rango de normalidad, pero, fueron menores a la puntuación $\mathrm{Z}=0$ (cero) de la tabla de referencia utilizada por el Ministerio de Salud Pública del Ecuador (2011). En cada subgrupo etario, los varones presentaron valores promedio de peso, talla e IMC mayores que las mujeres; excepto el de la talla en los grupos de 36-47 y 48-59 meses. Las diferencias de las medidas antropométricas según sexo fueron significativas, solamente, para: peso y talla en el grupo de 12-23 meses; peso e IMC en el subgrupo de 24-35 meses. 
Según la CEPAL, en menores de cinco años, el déficit de peso/edad (desnutrición global) en el Ecuador, desde 1986 hasta el 2006, fue más alta que en Bolivia, Paraguay y Perú; ventajosamente mantuvo una tendencia a la disminución, se redujo en 7.9\%; y logró llegar al 8,6\% en el año 2006 (Martínez \& Fernández, 2009). Siete años después, el estudio presente detectó una defíciencia de peso/edad en $8.2 \%$ en los niños de 12 a 59 meses; lo que demostraría un estancamiento, y aunque la desnutrición global ya no representa uno de los grandes problemas de salud pública a nivel nacional, supera la frecuencia de otros países latinoamericanos como Perú (3.4\% en 2012), Colombia (3.4\%, 2010) y Bolivia (4.5\% en 2008) (Martínez \& Palma, 2014); así como la prevalencia considerada como normal (2.5\%) (Martínez \& Fernández, 2009).

Respecto al déficit de talla/edad en menores de cinco años, su frecuencia se redujo en un $6.7 \%$ entre 1992 y 2006, determinándose una prevalencia de 25.8\% según la información de la Encuesta de Condiciones de Vida 2005-2006 (Martínez \& Palma, 2014). La UNICEF afirma que al menos uno de cada cinco niños ecuatorianos menores de cinco años tiene baja talla/edad (desnutrición crónica) (UNICEF, PMA Y OMS, en línea). Freire et al. (2013) en base a la Encuesta Nacional de Salud y Nutrición (ENSANUT-ECU) realizada en el 2012, calcularon que el 25.3\% de los preescolares presentaron retardo de la talla/edad. En la presente investigación, la baja talla/edad fue de $20.8 \%$ y la talla baja severa $3.5 \%$; de manera que coincidió con la información proporcionada por la UNICEF (en línea) y Freire et al. (2013); a la vez que se evidenció que desde el 2006, prácticamente, no se ha modificado la frecuencia. Por lo tanto, la persistencia de una alta prevalencia de talla menor a la normal continúa siendo un problema de salud pública; el mismo que es compartido por otros países de la región como Perú, que reportó en el 2010, 20.6\% de talla baja y 1.8 de talla baja severa en menores de cinco años (Molina, 2010).

A los problemas aún no resueltos de déficit de peso/edad y en especial de déficit de talla/edad; se han sumado el sobrepeso y la obesidad. De modo que a nivel del país se ha incrementado la prevalencia de sobrepeso y obesidad desde $4.2 \%$ en 1986 a 8.6 en 2012 (Freire et al., 2013). La presente investigación, realizada en el 2013 identificó, únicamente, a un 4.0\% de prescolares con sobrepeso y un $0.7 \%$ con obesidad; además de $16.3 \%$ con riesgo de sobrepeso; este porcentaje fue similar (16.8\%) al reportado por Freire et al. (2013).

Si se comparan los resultados del presente estudio con los datos obtenidos en las distintas encuestas nutricionales realizadas en el país a partir de 1986 (Freire et al., 2013; Informe final ENDEMAIN, 2014), se observa que la prevalencia de déficit de peso/edad tuvo una tendencia levemente decreciente y se ha reducido en alrededor de 4 puntos porcentuales a lo largo de 27 años; pero, el bajo peso severo se incrementó con respecto al año 2004. El déficit de talla/edad se ha reducido en alrededor de 16 puntos porcentuales, especialmente a expensas de la disminución de la talla baja (Informe final ENDEMAIN, 2014); a pesar de ser el indicador de condición nutricional que más mejoró en estas tres últimas décadas, continúa afectando a uno de cada cuatro niños. Afortunadamente se registró un incremento mínimo de sobrepeso/obesidad con respecto a 1996; pero, el riesgo de sobrepeso está alrededor del $16 \%$. Tomando en consideración el valor de referencia internacional que indica que un 2,3\% de niños en una población está normalmente ubicada debajo de 2 DE (Informe final ENDEMAIN, 2014), se puede concluir que el déficit de peso y principalmente el déficit de talla son problemas de salud presentes en la población estudiada.

En el Ecuador al igual que en otros países latinoamericanos como México (Bacardi-Gascón et al., 2013), Perú (Molina, 2010) y Argentina (Durán et al., 2009), los datos revelan la coexistencia de los problemas de déficit y exceso nutricional, demostrando la doble carga de la malnutrición como nuevo perfil epidemiológico. El análisis antropométrico desde la perspectiva de sexo, nos muestra que los niños presentaron mayor frecuencia de peso bajo severo, talla baja, talla baja severa, riesgo de sobrepeso y sobrepeso que las niñas. La mayor tendencia del sexo masculino al sobrepeso y a la obesidad ya se manifestó en la Encuesta Nacional de Salud y Nutrición 2011-2013 (Freire et al., 2013). El mejoramiento de las condiciones de vida de los países latinoamericanos en los últimos años, no parece haber tenido un efecto sustancial en las condiciones nutricionales en los niños de 12 a 59 meses de edad que asistieron para su control a los servicios de salud del eje urbano y área de influencia de la ciudad de Cuenca-Ecuador durante el año 2013. Afirmación que coincide con la de Martínez \& Palma (2014) respecto a que en América Latina persisten elevados índices de subnutrición y desnutrición crónica infantil, a los que se ha sumado el sobrepeso y la obesidad. 
Las correlaciones antropométricas peso/edad, talla/edad y peso/talla fueron directas, positivas y altas; sin embargo, expresaron valores menores a los obtenidos por un estudio realizado a preescolares, en Mérida, en 1996 (Angarita et al., 2001). La correlación IMC/edad fue inversa y negativa, con una distribución similar a la de la gráfica de IMC/edad utilizada por el MSP del Ecuador (2011).

\section{CONCLUSIONES}

En los preescolares de 12 a 59 meses que asistieron a control en los servicios de salud del eje urbano y área de influencia de la ciudad de Cuenca, se encontró que el estado nutricional fue normal según la relación peso/edad en $90.8 \%$, según la relación talla/edad en $75.6 \%$ y según la relación IMC/edad en $77.5 \%$. En $8.2 \%$ el peso/edad fue inferior al normal, en $24.3 \%$ la talla/edad fue menor a la normal, el $16.3 \%$ presentó riesgo de sobrepeso, $4.7 \%$ adoleció de sobrepeso/obesidad y 1.6 estuvo con emaciación o emaciación severa. El peso, la talla e IMC promedio fueron mayores en los niños que en las niñas. En los dos sexos el peso y la talla se incrementaron progresivamente con la edad, mientras que el índice de masa corporal disminuyó. Los varones presentaron mayor frecuencia de peso bajo severo, peso bajo, talla baja severa, talla baja, riesgo de sobrepeso y sobrepeso. Es decir que estuvo presente la malnutrición tanto por defecto como por exceso; y el problema nutricional más importante fue el déficit de talla/edad. Las correlaciones peso/edad, talla/edad y peso/talla fueron positivas y altas; en tanto que, la correlación del IMC/edad fue inversa y baja. Los niveles de correlación fueron mayores en las mujeres. Según los resultados obtenidos en el presente estudio, el mejoramiento de las condiciones de vida de los países latinoamericanos no evidenció una mejora sustancial en las condiciones nutricionales de los niños estudiados. Por lo tanto, las entidades de salud deberían establecer estrategias nuevas para mejorar las condiciones nutricionales de los niños y niñas preescolares. A la vez, se debería continuar realizando estudios transversales, prospectivos y periódicos cada cinco a 10 años sobre antropometría y condición nutricional de los niños y las niñas menores de cinco años con el fin de establecer los avances e introducir a tiempo estrategias innovadoras y oportunas.

\section{AGRADECIMIENTOS}

Las autoras expresan su profundo agradecimiento a los niños que participaron en el estudio y a sus madres, al personal que laboró en los 18 centros de salud pública del eje urbano y área de influencia inmediata de la ciudad de Cuenca: Centro de Salud $\mathrm{N}^{\circ} 1$ (Pumapungo), Paraíso, Machángara, Totoracocha, Centro de Salud No 2 (José María Astudillo Regalado), Terminal Terrestre, Barrial Blanco, Uncovía, Cebollar, San Pedro del Cebollar, Centro de Salud No 3 (Nicanor Merchán), Tomebamba, Turi, Centro de Salud No 4 (Carlos Elizalde), Baños, Virgen del Milagro, San Joaquín, Sayausí durante el año 2013, así como a las autoridades de salud de la provincia del Azuay porque hicieron posible la presente investigación.

\section{BIBLIOGRAFÍA}

Angarita, C., D. Machado, G. Morales, G. García de Méndez, F. Arteaga de Viloria, T. Silva, O.M. Alarcón C., 2001. Estado nutricional, antropométrico, bioquímico y clínico en preescolares de la comunidad rural de Canaguá. Estado Mérida. An. Venez. Nutr., 14(2), 75-85.

Arroyo, M., M. Freire, L. Ansotegui, A. Rocandio, 2010. Error intraobservador asociado con mediciones antropométricas realizadas por dietistas. Nutr. Hosp., 25(6), 1053-6.

Atalah, E. 2007. Una nueva referencia internacional de crecimiento infantil. Rev. Chil. Pediatr., 78(2), $186-92$. 
Bacardí-Gascón, M., E. Jones, A. Jiménez-Cruz, 2013. La prevalencia de la obesidad y la obesidad abdominal de los niños de cuatro a 16 años de edad que viven en la frontera entre México y EE.UU. Nutr. Hosp., 28(2), 479-485.

Cossio-Bolaños, M., M. Arruda, V. Núñez, J. Lancho, 2011. Efectos de la altitud sobre el crecimiento físico en niños y adolescentes. Rev. Andal. Med., 4(2), 71-6.

Crecimiento y desarrollo, 2003. Cuad. Hist. Salud Pública, (93). Disponible en: http://scielo.sld.cu/scielo.php?script=sci_arttext\&pid=S0045-91782003000100009\&lng=es el 20 de agosto de 2014.

Durán, P., G. Mangialavori, A. Biglieri, L. Kogan, E. Abeyá, 2009. Estudio descriptivo de la situación nutricional en niños de 6-72 meses de la República Argentina. Resultados de la Encuesta Nacional de Nutrición y Salud (ENNyS). Arch. Argent. Pediatr., 107(5), 397-404.

ENDEMAIN, 2014. Ecuador - Encuesta Demográfica y de Salud Materna e Infantil 2004, v01_M. Centro de Estudios de Población y Desarrollo Social (CEPAR). Descargado de http://microdata.worldbank.org/index.php/catalog/979 el 10 de septiembre de 2015.

ENSANUT-ECU 2011-2013. Ministerio de Salud Pública/Instituto Nacional de Estadística y Censos. Quito, Ecuador. Descargado de www.unicef.org/ecuador/ENSANUT_2011-2013_tomo_1.pdf el 09 de septiembre de 2015.

Freire, W.B., M.J. Ramírez, P. Belmont, M.J. Mendieta, K.M. Silva, N. Romero, K. Sáenz, P. Piñeros, L.F. Gómez, R. Monge, 2013. Resumen Ejecutivo. Tomo 1. Encuesta Nacional de Salud y Nutrición del Ecuador. Disponible en http://www.unicef.org/ecuador/ENSANUT_20112013_tomo_1.pdf.

Gutiérrez-Gómez, Y., J. Kain, R. Uauy, M. Galván, C. Corvalán C., 2009. Estado nutricional de preescolares asistentes a la Junta Nacional de Jardínes Infantiles de Chile: Evaluación de la concordancia entre indicadores antropométricos de obesidad y obesidad central. ALAN, 59(1), 30-37.

INEC, 2013. Día mundial de la población. Ecuador, Quito: INEC, Infografía. Descargado de http://www.ecuadorencifras.gob.ec/wp-content/uploads/downloads/2014/05/ DiaMundialPoblacion.jpg el 25 de julio de 2014.

León, A. D. Coromoto, P. Ledo, Y. Jiménez, M. Zavala, E. Díaz, 2010. Evaluación antropométrica de los pre-escolares del municipio Monseñor Iturriza, parroquia Tocuyo de la Costa del estado Falcón mayo-junio 2010. Rev. Electrónica de Portales Médicos.com. Descargado de http://www.salamandra.edu.co/fileadmin/documentos/articulos_academicos/pre-escolar.pdf el 20 de agosto de 2014.

Martínez, R., A. Fernández. 2006. Desnutrición infantil en América Latina y el Caribe. CEPALUNICEF. Desafíos 2:5-9. Descargado de www.unicef.org/lac/Desafiosnutricion(13).pdf el 12 de septiembre de 2015.

Martínez, R., A. Fernández, 2009. El costo del hambre. Impacto social y económico de la desnutrición infantil en el Estado Plurinacional de Bolivia, el Ecuador, Paraguay y el Perú. Santiago de Chile: CEPAL. Descargado de www.cepqal.org/publicaciones/xml/8/38538/dp-ddscosto-hambre.pdf el 25 de julio de 2014.

Martínez, R., A. Palma, 2014. Seguridad alimentaria y nutricional en cuatro países andinos. Una propuesta de seguimiento y análisis. CEPAL-Serie Políticas Sociales, 187. Santiago de Chile: Naciones Unidas. Descargado de http://www10.iadb.org/intal/intalcdi/PE/2014/14427.pdf el 28 de julio de 2014.

Molina, E., 2010. Evaluación del estado nutricional antropométrico de niños, niñas menores de 5 años y gestantes. Renut., 4(14), 705-714.

MSP-Ecuador, 2011. Normas de Atención Integral a la Niñez. Descargado de https://aplicaciones.msp.gob.ec/salud/archivosdigitales/documentosDirecciones/dnn/archivos/NO RMAS\%20DE\%20ATENCI\%C3\%93N\%20INTEGRAL\%20A\%20LA\%20NI\%C3\%91EZ\%202 011.pdf el 25 de julio de 2014. 
OMS, 2008. Curso de Capacitación sobre la Evaluación del Crecimiento del Niño. Patrones de Crecimiento del Niño de la OMS. Midiendo el Crecimiento de un Niño. Ginebra: Organización Mundial de la Salud. Descargado de http://www.who.int/childgrowth/training/es/ el 10 de septiembre de 2015.

UNICEF, PMA y OMS trabajan juntos contra la desnutrición infantil. Ecuador: UNICEF. Descargado de www.unicef.org/ecuador/media_9001.htm el 09 de septiembre de 2015.

Velásquez, M., G. Salazar, F. Vio, N. Díaz, A. Anziani, 2008. Validación de ecuaciones antropométricas para evaluar composición corporal en niños preescolares chilenos. Rev. Méd. Chil., 136(4), 433-441. 